\title{
Expression of YB-1 enhances production of murine leukemia virus vectors by stabilizing genomic viral RNA
}

\author{
Wei $\mathrm{Li}^{1,2}$, Xinlu Wang ${ }^{1}$, Guangxia Gao ${ }^{1 凹}$ \\ ${ }^{1}$ Key Laboratory of Infection and Immunity, Institute of Biophysics, Chinese Academy of Sciences, Beijing 100101, China \\ ${ }^{2}$ University of Chinese Academy of Sciences, Beijing 100049, China \\ $\triangle$ Correspondence: gaogx@moon.ibp.ac.cn \\ Received August 27, 2012 Accepted September 4, 2012
}

\begin{abstract}
Murine leukemia virus (MLV)-based retroviral vectors is widely used for gene transfer and basic research, and production of high-titer retroviral vectors is very important. Here we report that expression of the Y-box binding protein 1 (YB-1) enhanced the production of infectious MLV vectors. YB-1 specifically increased the stability of viral genomic RNA in virus-producing cells, and thus increasing viral RNA levels in both producer cells and virion particles. The viral element responsive to $Y B-1$ was mapped to the repeat sequence (R region) in MLV genomic RNA. These results identified YB-1 as a MLV mRNA stabilizer, which can be used for improving production of MLV vectors.
\end{abstract}

KEYWORDS Y-box binding protein 1, retroviral vector, RNA stability

\section{INTRODUCTION}

Murine leukemia virus (MLV)-based retroviral vectors have been widely used in clinical gene transfer (Palu et al., 2000; McTaggart and Al-Rubeai, 2002) and basic research. Unlike many of the other types of vectors currently used, retroviral vectors are able to stably introduce target genes into host cells without perturbing cell growth (Kim et al., 2000). However, the significant shortage is the low virus titer (Merten, 2004). To improve the titer of infectious viral vectors, extensive efforts have been made to optimize the condition or parameters in viral production, such as the choice of the producer cell lines, medium additives, serum and the type of bioreactors (Merten, 2004).

The life cycle of retroviruses is comprised of multiple steps, involving many host factors (Goff, 2007). Steps for production of virion particles include viral RNA transcription, processing, translation, virus assembly and budding. Interactions between host factors and viral proteins or RNAs play important regulatory roles in this process. For example, it has been reported that expression of IMP1 enhanced production of MLV vectors by facilitating viral genomic RNA packaging (Mai and Gao, 2010).

Y-box binding protein 1 (YB-1) is a member of protein family with a conserved cold domain (Wolffe et al., 1992). It was originally identified as a transcription factor that recognizes the Y-box motif in HLA class II gene promoters (Didier et al., 1988). YB-1 binds to DNA and RNA, and is involved in many steps of nucleic acid biogenesis, including DNA replication and repair, mRNA transcription, pre-mRNA splicing, mRNA stability and translation processing (Wilkinson and Shyu, 2001; Skalweit et al., 2003; Berquin et al., 2005; Evdokimova et al., 2006; Eliseeva et al., 2011). For example, YB-1 can either stimulate or inhibit transcription of many cellular and viral genes (Kohno et al., 2003; Eliseeva et al., 2011). It has been reported that YB-1 bound to the GC-rich motif of HIV-1 5'LTR and activated the transcription of HIV-1 promoter (Sawaya et al., 1998). YB-1 also recognizes specific sequence in some mRNAs and stabilizes mRNAs including renin, IL-2, VEGF and GM-CSF (Eliseeva et al., 2011).

In the present study, we investigated whether YB-1 is able to promote the production of MLV vectors. We provide evidence indicating that expression of YB-1 facilitates $M L V$-based retroviral vector production by stabilizing viral genomic RNA. 


\section{RESULTS}

\section{Overexpression of YB-1 increased retroviral vector production}

MLV-luc is a retroviral vector carrying a firefly luciferase reporter (Gao et al., 2002; Guo et al., 2004). To produce VSV_G pseudotyped MLV-luc vector, pMLV-luc was cotransfected into HEK293T cells with pVSVG, a plasmid expressing VSV_G, and pHIT60, a plasmid expressing MLV proteins. A plasmid expressing Flag-tagged YB-1 was transfected into 293T cells together with MLV-luc constructs. A plasmid expressing renilla luciferase was also included as a control for transfection efficiency and sample handing. The produced retroviral vectors were used to infect recipient cells, and the firefly luciferase activity in the recipient cells normalized by renilla luciferase activity in the producer cells was used as an indicator for virus production. Data show that the firefly luciferase activity in the recipient cells infected with MLV-luc vectors produced in the presence of YB-1 was much higher than that in the presence of empty vectors (Fig. 1B), suggesting that YB-1expression improved MLV-luc produc-

A

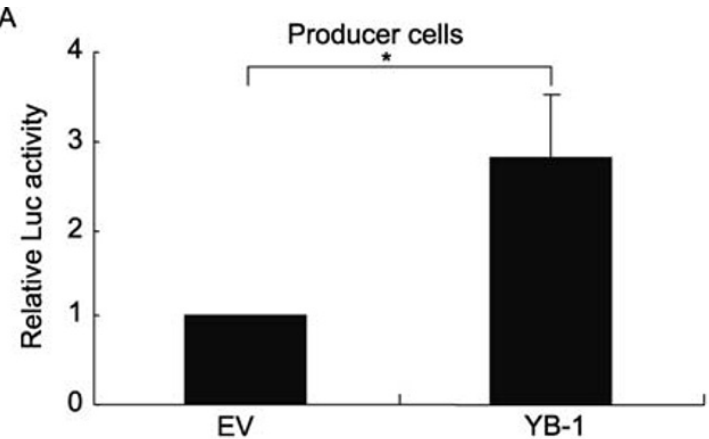

C

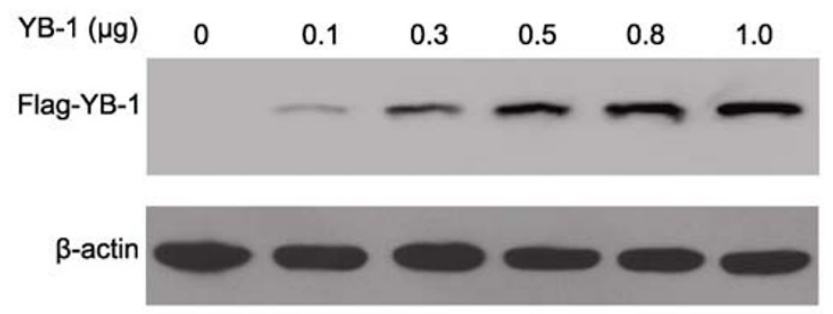

tion. In the producer cells, YB-1 expression also increased the firefly luciferase activity (Fig. 1A), implicating that YB-1 increased MLV-luc expression.

To test whether YB-1 increases MLV-luc production in a dose-dependent manner, increasing amounts of the YB-1-expressing plasmid was co-transfected with the MLV-luc producing constructs. With increasing expression levels of YB-1 (Fig. 1C), the firefly luciferase activity increased in recipient cells at low dosages of YB-1 (Fig. 1D). However, at high dosages of YB-1, the stimulatory effect dropped (Fig. 1D). These results established that YB-1 enhanced MLV-luc production in an YB-1 dose dependent manner.

\section{YB-1 increased viral genomic RNA levels in both virion particles and producer cells}

We next analyzed viral genomic RNA levels in the producer cells and virion particles. To analyze viral genomic RNA levels in the virion particles, MLV-luc pseudovirus produced in the presence of empty vector or YB-1 were purified and concentrated by ultracentrifugation. Viral genomic RNA was

B

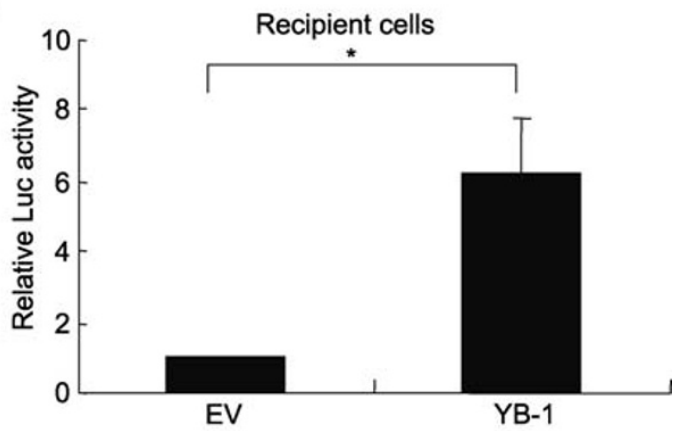

D

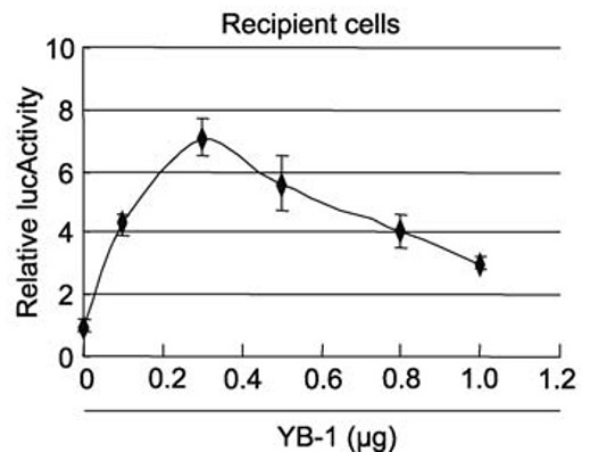

Figure 1. Overexpression of YB-1 in producer cells increased the production of retroviral vector MLV-luc. MLV-luc-producing constructs pCMV-VSVG, pHIT60 and pMLV-Luc in a total of $1.8 \mu \mathrm{g}$ at the ratio of 1.3:1.3:1, and $0.01 \mu \mathrm{g}$ of pRL-TK, a plasmid expressing renilla luciferase, were cotransfected into HEK293T cells in $6 \mathrm{~cm}$ dishes together with $0.2 \mu \mathrm{g}$ of empty vector, pCMV-HA-Flag-YB-1 (A and $B$ ), or with the indicated amounts of YB-1 (C and D). At $48 \mathrm{~h}$ post-transfection, virus was harvested to infect HEK293 cells and luciferase activities in the producer cells were measured. At $48 \mathrm{~h}$ postinfection, luciferase activity was measured in the recipient cells. (A) Firefly luciferase activity normalized by renilla luciferase activity in the producer cells. (B and D) Firefly luciferase activity in the recipient cells normalized by renilla luciferase activity in the producer cells. (C) Proteins indicated in the producer cells were detected by Western blotting. The data are means \pm SD of three independent measurements. *denotes $P<0.05$. 
extracted from the virions. To help sample handling, total RNA of naive HEK293 cells was added to each sample before RNA-extraction. Viral genomic RNA levels were analyzed by RT-qPCR. Data show that the RNA level in the virions from YB-1-expressing cells was significantly higher than that produced in the control cells (Fig. 2A). Total RNAs of producer cells were also extracted, and subjected to Northern blotting to measure viral genomic RNA levels. Expression of YB-1 significantly increased the level of viral genomic RNA in the producer cells (Fig. 2B). These results established that YB-1 enhanced viral genomic RNA levels in both virion particles and producer cells.

\section{YB-1 stabilized viral genomic RNA in producer cells}

YB-1 has been reported as an RNA stabilizer for some mRNAs (Evdokimova et al., 2001; Nekrasov et al., 2003; Eliseeva et al., 2011). The above results suggest that the increased viral RNA levels in producer cells may result from improved RNA stability. To test this possibility, the decay rates of MLV-luc genomic RNA were analyzed in the presence and absence of YB-1 overexpression. Transcription of RNA was blocked by addition of actinomycin D, and total RNAs were extracted at different time points. MLV-luc genomic RNA level were assayed by Northern Blot. Indeed, the half-life of MLV-luc genomic RNA in the YB-1-expressing cells was significantly longer than that in the control cells (Fig. 3). In contrast, YB-1 had little effect on the stability of GAPDH

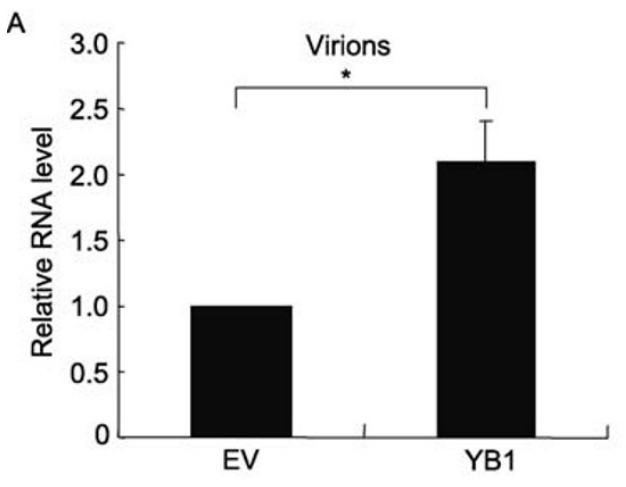

mRNA. These results implicate that YB-1 expression stabilized viral genomic RNA in viral producer cells.

\section{The $R$ region of MLV UTR is required for its sensitivity to YB-1}

YB-1 specifically stabilized viral genomic RNA of MLV, suggesting that some specific sequences may exist in the viral RNA to mediate its response to YB-1. To map the responsive sequence in MLV-luc RNA, the $5^{\prime} L T R$ and $3^{\prime} L T R$ of MLV-luc were cloned into pGI3-luc reporter to assay their abilities to confer sensitivity to YB-1 (Fig. 4A). The constructs were transfected into HEK293 cells in the presence or absence of YB-1. A plasmid expressing renilla luciferase, pRL-TK, was included to serve as a control for transfection efficiency and sample handing. Data show that both the 5'UTR and $3^{\prime} U T R$ rendered the reporter sensitive toYB-1 (Fig. 4B). The mRNA transcribed from the 5'UTR contains U5 and R sequences, while the mRNA transcribed from Luc-3'UTR contains U3 and $R$ sequences (Fig. 4A). These results suggest that the $R$ region, which is common in both reporters, may be the responsive element. To test this possibility, the $\mathrm{R}$ region or U5 was cloned upstream of luciferase coding sequence (Fig. 4A). Indeed, the $\mathrm{R}$ sequence rendered the reporter sensitive to the stimulatory effect of YB-1 (Fig. 4C). In contrast, the U5 sequence failed to do so (Fig. 4C). These results indicate $R$ region of MLV is the responsive element in MLV to the stimulatory effect of YB-1.

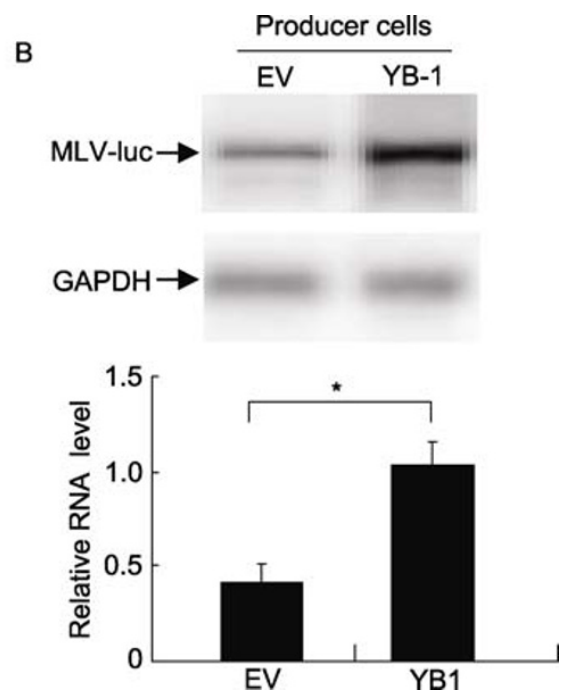

Figure 2. YB1 overexpression increased viral genomic RNA levels in both virions and producer cells. MLV-luc-producing constructs were cotransfected into HEK293T cells with empty vector or pCMV-HA-Flag-YB-1. (A) At $48 \mathrm{~h}$ post-transfection, supernatants were collected and purified. Viral genomic RNA was isolated by Trizol extraction. Ten $\mu \mathrm{g}$ of the total RNA of naïve HEK293 cells was added to each sample before RNA-extraction. Viral genomic RNA levels were measured by quantitative RT-qPCR with primers for luciferase gene. GAPDH from the added cellular RNA was used as an internal control. Data presented are means \pm SD from three independent measurements. *denotes $P<0.05$. (B) The producer cells were lyzed in Trizol buffer for total RNA extraction. The levels of viral genomic RNA were analyzed by Northern Blot and measured using Phosphoimager. GAPDH mRNA was used as an internal control. Data presented are means \pm SD of from two independent measurements. ${ }^{*}$ denotes $P<0.05$. 
A

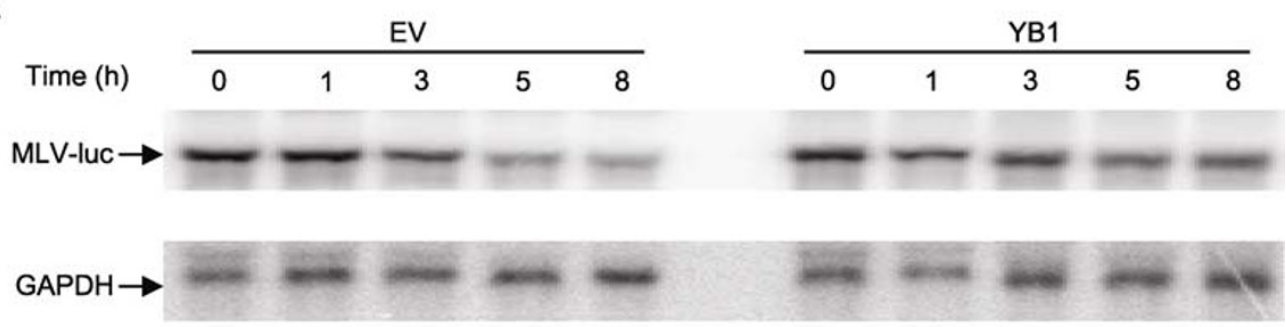

B

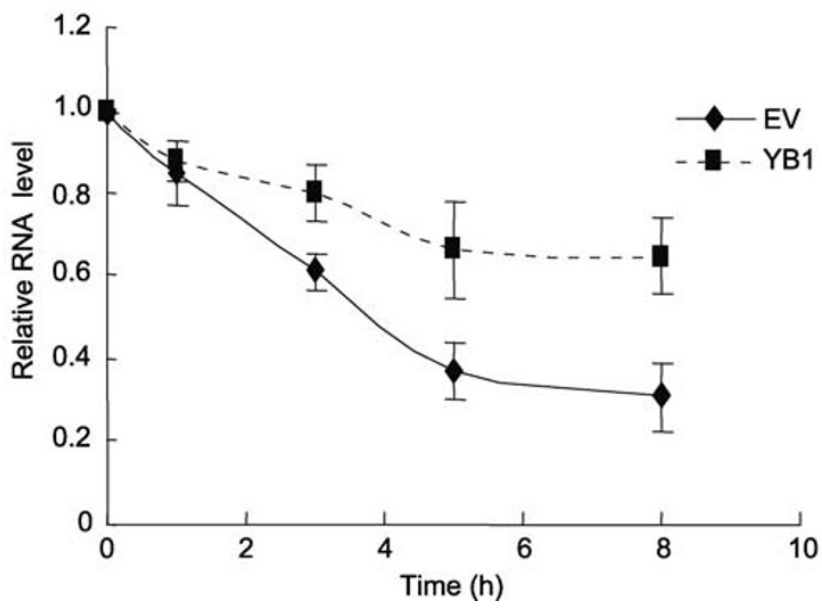

Figure 3. YB-1 stabilized the viral genomic RNA in producer cells. pMLV-Luc was cotransfected with empty vector or pCMV-HA-Flag-YB-1 into HEK293T cells. At $24 \mathrm{~h}$ post-transfection, cells were equally divided into 5 dishes and $12 \mathrm{~h}$ later actinomycin D was added to block transcription. At time points indicated, cells were lysed and RNA was extracted and subjected to Northern blotting analysis (A). Relative levels of viral RNA were quantitated using Phosphoimager and normalized with that of GAPDH mRNA (B). Data presented are means \pm SD from two independent measurements.

\section{DISCUSSION}

Retroviral vectors produced by DNA transfection of producer cells usually contain a large amount of non-infectious virus. The ratio of infectious virions to the total MLV particles could be as low as 1/100 (Higashikawa and Chang, 2001). In this report, we demonstrate that overexpression of YB-1 increased the production of infectious MLV vector (Fig. 1). Further analyses revealed that YB-1 increased the genomic RNA levels in both producer cells and virion particles (Fig. 2). Increased viral RNA level by YB-1 in producer cells is likely to provide more genomic RNA for packaging into virions, thus promoting the titer of infectious virus particles.

We further demonstrated that YB-1 stabilized the genomic RNA in producer cells (Fig. 3). YB-1 has been reported to regulate a variety of DNA- and RNA-dependent processes (Eliseeva et al., 2011). It awaits further investigation whether YB-1 also promotes MLV vector production by regulating other $\mathrm{mRNA}$ related processes, such as $\mathrm{mRNA}$ transcription, processing, transport and mRNA localization.

YB-1 can stabilize mRNA in two ways. First, YB-1 forms complexes with mRNAs at a high YB-1/mRNA ratio in which the 5'-and 3'-termini of RNA molecules are buried inside protein globules and are thus inaccessible to exoribonucleases, resulting in general stabilization of mRNA. Second, YB-1 recognizes specific sequences and thus selectively protects mRNAs from degradation (Eliseeva et al., 2011). For example, in complex with other proteins, YB-1 specifically binds to CU-rich elements in the 3'UTR of renin mRNA, and both 5'UTR and 3'UTR of VEGF mRNA for their stabilization (Skalweit et al., 2003; Coles et al., 2004). YB-1 has also been reported to interact with the AU-rich elements in the 3'UTR of GM-CSF mRNA to increase the stability of the mRNA (Esnault and Malter, 2003). For MLV, we here identified the $R$ region as a responsive element to YB-1-mediated specific stabilization (Fig. 4). These results provide an additional example of YB-1-mediated stabilization of specific mRNAs.

In conclusion, we identified YB-1 as a MLV mRNA stabilizer. Expression of YB-1 increased viral RNA levels in both producer cells and virion particles, and thus the titer of infectious virion particles. YB-1 can be used for improving production of MLV vectors. 
A

\begin{tabular}{c|c|c|c|}
5 & 5 LTR \\
MLV-Luc & U3 & $\mathrm{R}$ & $\mathrm{U} 5$ \\
\cline { 2 - 6 } \\
\hline
\end{tabular}

pGL3-luc

\begin{tabular}{|l|c|}
\hline Psv40 & Luciferase \\
\hline
\end{tabular}

\begin{tabular}{|l|l|l|l|} 
5'LTR-Luc U3 & R & U5 & Luciferase \\
\hline
\end{tabular}

Luc-3'LTR

\begin{tabular}{|l|l|l|l|l|}
\hline Psv40 & Luciferase & U3 & R & U5 \\
\hline \multicolumn{1}{|l}{} \\
\cline { 2 - 3 } \\
\cline { 2 - 3 }
\end{tabular}

\begin{tabular}{c|c|c|c|}
\hline R-Luc & Psv40 & $\mathrm{R}$ & Luciferase \\
\hline \multicolumn{2}{c}{. } \\
\hline
\end{tabular}

U5-Luc \begin{tabular}{|l|l|l|}
\hline Psv40 & U5 & Luciferase \\
\hline \multicolumn{2}{c}{} \\
\hline
\end{tabular}
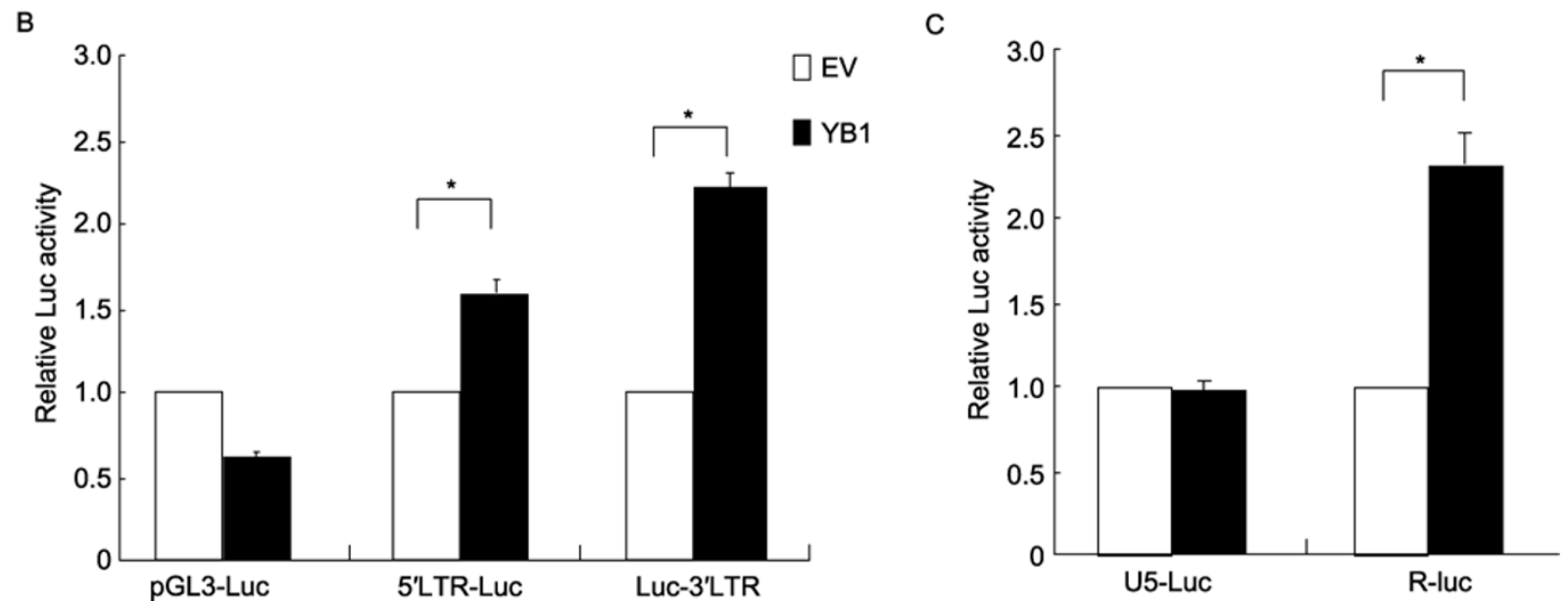

Figure 4. Mapping of sequences in MLV responsive to YB-1. (A) Schematic representation of the reporter constructs. Expected mRNAs transcribed from these constructs are shown under each construct. ( $B$ and $C$ ) Reporter constructs were cotransfected to HEK293T cells with empty vector or pCMV-HA-Flag-YB-1. A renilla luciferase-expressing plasmid, pRL-TK, was included. At $48 \mathrm{~h}$ post-transfection, cells were lysed and luciferase activities were measured. Firefly luciferase activity was normalized with renilla luciferase activity. Data presented are means \pm SD of three independent measurements. ${ }^{*}$ denotes $P<0.05$.

\section{MATERIALS AND METHODS}

\section{Plasmids}

MLV-luc producing plasmids pCMV-VSVG, pHIT60 and pMA-Luc have been previously described (Gao et al., 2002; Guo et al., 2004).

pCMV-HA-Flag-YB-1 expresses Flag-tagged YB-1.The sequence encoding YB-1 was PCR-amplified from a human cDNA library and cloned into pCMV-HF (Guo et al., 2007). The primers are listed below, with the built-in restriction sites underlined.

YB-1FP: ATGCGGCCGCTATGAGCAGCGAGGCCGAGA

YB-1RP: TA GCGGCCGCTTACTCAGCCCCGCCCTGC

Reporters pGI3-5'LTR and pGI3-3'UTR, which contain the 5'LTR and $3^{\prime} L T R$ of MLV, respectively, have been reported previously (Guo et al., 2004). Reporter pcDNA4-luc was constructed by inserting the coding sequence of firefly luciferase into pcDNA4/to/myc-HisB (Invitrogen) using the Xhol and Sacll sites. Plasmids pcDNA4-R-luc and pcDNA4-U5-luc contain the R and U5 sequences of MLV, respectively. To generate pcDNA4-R-luc, pairs of oligonucleotides (R-F and $\mathrm{R}-\mathrm{R}$ ) of the $\mathrm{R}$ sequences were annealed and cloned into pcDNA4-luc using BamHI and $\mathrm{Xhol}$ sites. The same strategy was used to generate pcDNA4-U5-luc. Sequences of the primers are listed below.

R-F: 5'-GATCCGCGCCAGTCCTCCGATTGACTGAGTCGCCCGGGTACCCGTGTATCCAATAAACCCTCTTGCAGTTGCAGCCACCATGGCC-3'

R-R: 5'-TCGAGGCCATGGTGGCTGCAACTGCAAGAGGGTTTATTGGATACACGGGTACCCGGGCGACTCAGTCAATCGGAGGACTGGCGCG-3' 
U5-F: 5'-GATCCTCCGACTTGTGGTCTCGCTGTTCCTTGGGAGGGTCTCCTCTGAGTGATTGACTACCCGTCAGCGGGGGTCTTTCAGCCACCATGGCC-3'

U5-R: 5'-TCGAGGCCATGGTGGCTGAAAGACCCCCGCTGACGGGTAGTCAATCACTCAGAGGAGACCCTCCCAAGGAACAGCGAGACCACAAGTCGGAG-3'

\section{Cell culture and viral infection}

HEK293 cells (ATCC CRL-1573) and HEK293T cells (ATCC CRL-11268) were maintained in DMEM supplemented with $1 \%$ antibiotics and $10 \%$ fetal bovine serum (Invitrogen).

To produce VSV_G pseudotyped MLV-luc vector, pMLV-luc was cotransfected into HEK293T cells with pVSVG, a plasmid expressing VSV_G, and pHIT60, a plasmid expressing MLV proteins. A plasmid expressing renilla luciferase was included as a control for transfection efficiency and sample handing. At $48 \mathrm{~h}$ post-transfection, the supernatants were collected and filtered through $0.45 \mu \mathrm{m}$ filters to remove cell debris. The virus-containing supernatants were used to infect HEK293 (recipient) cells. Luciferase activities were measured with the Dual-Luciferase Assay System (Promega).

To purify and concentrate virion particles, supernatants were first subjected to $25 \% / 45 \%$ sucrose step gradient centrifugation at 25,000 rpm with Hitachi P40ST rotor for $2 \mathrm{~h}$ at $4^{\circ} \mathrm{C}$ and then concentrated by ultracentrifugation with $25 \%$ sucrose cushion for $2 \mathrm{~h}$. The pellets were resuspended in TNE buffer $(50 \mathrm{mmol} / \mathrm{L}$ Tris- $\mathrm{HCl} \mathrm{pH} 7.5,100 \mathrm{mmol} / \mathrm{L}$ $\mathrm{NaCl}$ and $1 \mathrm{mmol} / \mathrm{L}$ EDTA) and stored at $-80^{\circ} \mathrm{C}$ for future use.

To evaluate the effect of YB-1 on reporter expression, pCMV-HA-Flag-YB-1 or Empty vector pCMV-HA-Flag was cotransfected with the reporter plasmid. A plasmid expressing renilla lucierase was included to serve as a control for transfection efficiency and sample handling. Cells were lysed and luciferase activities were measured with the Luciferase Assay System (Promega) at $48 \mathrm{~h}$ post-transfection.

\section{Measurement of the viral RNA levels}

The purified virions were lysed in Trizol buffer (Invitrogen). Ten $\mu \mathrm{g}$ of total RNA of HEK293T cells was added into each sample as a control for sample handling. To remove DNA contamination, RNAs were treated with the DNA Free kit (Ambion). Viral genomic RNA levels were measured by real time-PCR with primers specific the for the luciferase gene. The reaction conditions for real time PCR in Rotor-gene 6000 (Corbett Life Science) were 1) $50^{\circ} \mathrm{C} 2 \mathrm{~min}, 1$ cycle; 2) $\left.95^{\circ} \mathrm{C} 5 \mathrm{~min}, 1 \mathrm{cycle} ; 3\right) 95^{\circ} \mathrm{C} 15 \mathrm{~s}, \quad 60^{\circ} \mathrm{C} 30 \mathrm{~s}, 72^{\circ} \mathrm{C} 30 \mathrm{~s}, 40$ cycles; 4) $72^{\circ} \mathrm{C} 10 \mathrm{~min}, 1$ cycle. The mRNA level of gapdh served as an internal control. Sequences of the primers are listed below:

qLuc-FP: CCAGGGATTTCAGTCGATGT

qLuc-RP: AATCTGACGCAGGCAGTTCT

qGAPDH-FP: TCACTGCCACCCAGAAGACTGTGG

qGAPDH-RP: GGTCCACCACCCTGTTGCTGTAGCC

The RNA levels in producer cells were measured by Northern blotting analysis following the procedure described previously (Guo et al., 2007).

\section{ACKNOWLEDGMENTS}

This work was supported in part by Grants (to G.G.) from National
Natural Science Foundation of China (Grant Nos. 81030030, 81101262 and 81028011) and the National Basic Research Program (973 Program) (No. 2012CB910203).

\section{ABBREVIATIONS}

ARE, AU-rich element; LTR, long terminal repeat; HIV-1, human immunodeficiency virus type I; MLV, Moloney murine leukemia virus; UTR, untranslated regions; YB-1, Y-box binding protein 1

\section{REFERENCES}

Berquin, I.M., Pang, B., Dziubinski, M.L., Scott, L.M., Chen, Y.Q., Nolan, G.P., and Ethier, S.P. (2005). Y-box-binding protein 1 confers EGF independence to human mammary epithelial cells. Oncogene 24, 3177-3186.

Coles, L.S., Bartley, M.A., Bert, A., Hunter, J., Polyak, S., Diamond, P., Vadas, M.A., and Goodall, G.J. (2004). A multi-protein complex containing cold shock domain (Y-box) and polypyrimidine tract binding proteins forms on the vascular endothelial growth factor mRNA. Potential role in mRNA stabilization. Eur J Biochem 271, 648-660.

Didier, D.K., Schiffenbauer, J., Woulfe, S.L., Zacheis, M., and Schwartz, B.D. (1988). Characterization of the cDNA encoding a protein binding to the major histocompatibility complex class II $Y$ box. Proc Natl Acad Sci U S A 85, 7322-7326.

Eliseeva, I.A., Kim, E.R., Guryanov, S.G., Ovchinnikov, L.P., and Lyabin, D.N. (2011). Y-box-binding protein 1 (YB-1) and its functions. Biochemistry (Mosc) 76, 1402-1433.

Esnault, S., and Malter, J.S. (2003). Hyaluronic acid or TNF-alpha plus fibronectin triggers granulocyte macrophage-colonystimulating factor mRNA stabilization in eosinophils yet engages differential intracellular pathways and mRNA binding proteins. J Immunol 171, 6780-6787.

Evdokimova, V., Ovchinnikov, L.P., and Sorensen, P.H. (2006). Y-box binding protein 1: providing a new angle on translational regulation. Cell Cycle 5, 1143-1147.

Evdokimova, V., Ruzanov, P., Imataka, H., Raught, B., Svitkin, Y., Ovchinnikov, L.P., and Sonenberg, N. (2001). The major mRNA-associated protein YB-1 is a potent $5^{\prime}$ cap-dependent mRNA stabilizer. EMBO J 20, 5491-5502.

Gao, G., Guo, X., and Goff, S.P. (2002). Inhibition of retroviral RNA production by ZAP, a CCCH-type zinc finger protein. Science 297, 1703-1706.

Goff, S.P. (2007). Host factors exploited by retroviruses. Nat Rev Microbiol 5, 253-263.

Guo, X., Carroll, J.W., Macdonald, M.R., Goff, S.P., and Gao, G. (2004). The zinc finger antiviral protein directly binds to specific viral mRNAs through the $\mathrm{CCCH}$ zinc finger motifs. J Virol 78, 12781-12787.

Guo, X., Ma, J., Sun, J., and Gao, G. (2007). The zinc-finger antiviral protein recruits the RNA processing exosome to degrade the target mRNA. Proc Natl Acad Sci U S A 104, 151-156.

Higashikawa, F., and Chang, L. (2001). Kinetic analyses of stability of simple and complex retroviral vectors. Virology 280, 124-131.

Kim, S.H., Kim, S., and Robbins, P.D. (2000). Retroviral vectors. Adv Virus Res 55, 545-563. 
Kohno, K., Izumi, H., Uchiumi, T., Ashizuka, M., and Kuwano, M. (2003). The pleiotropic functions of the Y-box-binding protein, YB-1. Bioessays 25, 691-698.

Mai, Y., and Gao, G. (2010). Expression of IMP1 enhances production of murine leukemia virus vector by facilitating viral genomic RNA packaging. PLoS One 5, e15881.

McTaggart, S., and Al-Rubeai, M. (2002). Retroviral vectors for human gene delivery. Biotechnol Adv 20, 1-31.

Merten, O.W. (2004). State-of-the-art of the production of retroviral vectors. J Gene Med 6 Suppl 1, S105-124.

Nekrasov, M.P., Ivshina, M.P., Chernov, K.G., Kovrigina, E.A., Evdokimova, V.M., Thomas, A.A., Hershey, J.W., and Ovchinnikov, L.P. (2003). The mRNA-binding protein YB-1 (p50) prevents association of the eukaryotic initiation factor elF4G with mRNA and inhibits protein synthesis at the initiation stage. J Biol Chem 278, 13936-13943.
Palu, G., Parolin, C., Takeuchi, Y., and Pizzato, M. (2000). Progress with retroviral gene vectors. Rev Med Virol 10, 185-202.

Sawaya, B.E., Khalili, K., and Amini, S. (1998). Transcription of the human immunodeficiency virus type 1 (HIV-1) promoter in central nervous system cells: effect of YB-1 on expression of the HIV-1 long terminal repeat. J Gen Virol 79 (Pt 2), 239-246.

Skalweit, A., Doller, A., Huth, A., Kahne, T., Persson, P.B., and Thiele, B.J. (2003). Posttranscriptional control of renin synthesis: identification of proteins interacting with renin mRNA 3'-untranslated region. Circ Res 92, 419-427.

Wilkinson, M.F., and Shyu, A.B. (2001). Multifunctional regulatory proteins that control gene expression in both the nucleus and the cytoplasm. Bioessays 23, 775-787.

Wolffe, A.P., Tafuri, S., Ranjan, M., and Familari, M. (1992). The Y-box factors: a family of nucleic acid binding proteins conserved from Escherichia coli to man. New Biol 4, 290-298. 